\title{
Minireview
}

\section{Modifications of histone cores and tails in V(D)J recombination Kathrin Muegge}

Address: Laboratory of Molecular Immunoregulation, SAIC-Basic Science Program, National Cancer Institute, Frederick, MD 21701, USA. E-mail: muegge@ncifcrf.gov

Published: 3I March 2003

Genome Biology 2003, 4:211

The electronic version of this article is the complete one and can be found online at http://genomebiology.com/2003/4/4/2I I

(C) 2003 BioMed Central Ltd

\begin{abstract}
The organization of chromatin and modifications to the tails of histone proteins are thought to be important in regulating the rearrangement of $V, D$ and J gene segments, which encode immunoglobulins and T-cell receptors. A recent study shows that methylated lysine 79 in the core region of histone $\mathrm{H} 3$ also plays a role by providing a euchromatic 'mark' that may regulate access of the $\mathrm{V}(\mathrm{D}) \mathrm{J}$ recombinase.
\end{abstract}

The organization of DNA into chromatin allows dense packaging of the genetic material and protects it against damage. Chromatin packaging also allows functional organization of the genome into active regions of 'open' euchromatin, which are accessible to regulatory factors, and silent regions of condensed heterochromatin $[1,2]$. The reversible switch from euchromatin to heterochromatin provides a means of controlling processes occurring on the DNA, such as replication and transcription, and has been proposed - in the so-called 'accessibility hypothesis' - to be crucial for regulating V(D)J recombination during immune-system development [3].

During V(D)J recombination, gene segments that encode variable receptors within the immune system are recombined and newly assembled to allow expression of distinct immunoglobulins or T-cell receptors (TCRs) [4,5]. The $\mathrm{V}(\mathrm{D}) \mathrm{J}$ recombinase, a dimer of the site-specific recombination proteins RAG1 and RAG2, binds to recombination signal sequences that flank the $\mathrm{V}, \mathrm{D}$ and $\mathrm{J}$ gene segments and initiates the process of cleaving the DNA sequences that are to be rearranged. Because the same recombinase is present in both $\mathrm{T}$ and B cells, but only B cells fully rearrange their immunoglobulin loci and only T cells their TCR loci, it has been proposed that a specific modulation of chromatin structure might open up recombination signal sequences to provide access for the recombinase. This 'chromatin accessibility' model could explain lineage and allele specificity of recombination as well as the temporal order of V(D)J rearrangement during development. A recent study from the
Struhl and Oettinger laboratories [6] has provided new evidence in support of this model.

\section{Chromatin accessibility and V(D)J recombination}

The accessibility model gained support from the observation of close association between the processes of transcription and recombination of unrearranged V, D and $\mathrm{J}$ fragments. Deletion of cis-acting enhancer or promoter sequences frequently inhibited both processes [5,7], and it was proposed that similar molecular mechanisms might open up chromatin to allow access for both the transcriptional machinery and the RAG recombinase. Many of the modifications of heterochromatin or euchromatin and processes involving chromatin that are associated with transcriptional regulation have been considered as potential regulators of $\mathrm{V}(\mathrm{D}) \mathrm{J}$ recombination, including $\mathrm{CpG}$ methylation, posttranslational modifications of histone tails, for example by acetylation or methylation, and chromatin remodeling driven by ATP-dependent Swi/Snf protein complexes. Methylation of CpG motifs, a hallmark of heterochromatin, was the first of these to be demonstrated to inhibit V(D)J recombination [8], but its importance has been questioned since demethylation precedes rearrangement in some, but not all, TCR and immunoglobulin loci, and global demethylation is not sufficient for recombination [9].

Acetylation of histone tails, a key event in regulating transcriptional processes, was found to be closely associated 
with the ability of lymphocytes to recombine their $\mathrm{V}, \mathrm{D}$ and $\mathrm{J}$ segments [4,7]. Acetylation of histone $\mathrm{H}_{3}$ or histone $\mathrm{H}_{4}$ is elevated at gene segments that can recombine in a specific cell type and is reduced at segments that cannot. Moreover, hyperacetylation (induced, for example, by histone deacetylase inhibitors) rescued recombination defects caused by the elimination of enhancer elements or of extracellular signals that induce recombination. Furthermore, hyperacetylation can act in concert with ATP-driven chromatinremodeling complexes, such as Swi/Snf, to facilitate RAG-mediated cleavage of V(D)J DNA sequences in vitro. These results suggest that histone hyperacetylation precedes recombination by opening chromatin and promoting access for the recombinase.

\section{Modification of histone tails}

Although histone acetylation probably contributes to recombination, several studies $[10,11]$ have demonstrated that acetylation is not sufficient to grant recombination factors access to chromatin. Thus, other chromatin modifications must be required. There is methylation of distinct lysine residues of histones in euchromatin versus heterochromatin. Methylated lysine 4 marks euchromatin, and this mark is elevated within recombinationally active TCR loci (M.S. Krangel, personal communication), whereas methylation at lysine 9, a heterochromatic marker, is elevated in recombinationally silent regions [6], and methylated lysine 27 was recently proposed to play a role during recombination [12]. Deficiency of the histone methyltransferase Ezh2 (enhancer of Zeste 2) leads to reduced recombination at the most distal $\mathrm{V}$ gene segments of the immunoglobulin heavy chain $\left(\mathrm{V}_{\mathrm{H}} \mathrm{J} 558\right.$ gene cluster), suggesting that these distal V segments are regulated differently from the ones that are closer to the D and $\mathrm{J}$ segments. Absence of Ezh2 causes a global methylation defect of histone $\mathrm{H}_{3}$ that mainly affects methylation of lysine 27. The reduced recombination of distal V gene segments correlates with a specific decrease in histone $\mathrm{H}_{3}$ methylation at these gene segments, suggesting a functional role for methylated lysine 27 in controlling access by recombination factors. The specific recombination defect at the $\mathrm{V}_{\mathrm{H}} \mathrm{J}_{55} 8$ gene cluster in Ezh2-deficient cells is reminiscent of the one reported in cells lacking the receptor for the cytokine interleukin (IL) 7, suggesting a potential functional relationship between IL-7 and V(D)J recombination [13]. Indeed, IL-7 signaling enhances methylation of lysine 27 of histone $\mathrm{H}_{3}$, indicating that cytokine signaling regulates $\mathrm{V}(\mathrm{D}) \mathrm{J}$ recombination, in part, through the control of histone methylation.

\section{Modifications to core portions of histone $\mathrm{H} 3$}

The posttranslational histone modifications discussed so far occur within the amino-terminal histone tails, which protrude from the nucleosome core that is composed of an octamer of the four core histones $\mathrm{H}_{2} \mathrm{~A}, \mathrm{H}_{2} \mathrm{~B}, \mathrm{H}_{3}$ and $\mathrm{H}_{4}$ and
146 base-pairs of DNA wrapped around the histones. The 'histone code' theory [14] suggests that different combinations of histone modifications may form a code and thereby serve as platform for the recruitment of other chromatinremodeling activities. Because histone tails are exposed on the nucleosome surface, one can easily imagine how tail modifications could fit with this model. Over the last year, however, lysine 79, which is at the surface of the nucleosomal core, has also been identified as a site of histone $\mathrm{H}_{3}$ methylation $[15,16]$. New evidence from the Struhl and Oettinger laboratories [6] suggests that methylated lysine 79 acts as a general marker for active chromatin and may be involved in controlling access for recombination factors. Previously, the Struhl laboratory [17] had identified Dot1 as a histone methyltransferase that can methylate lysine 79 of histone $\mathrm{H}_{3}$. In the more recent study [6], the same group determined the methylation pattern of this residue in vivo using Saccharomyces cerevisiae as a model organism. Low levels of lysine 79 methylation were observed at heterochromatic regions, such as telomeric or mating-type loci. Spreading of heterochromatin caused by overexpression of Sir3 (silent information regulator), which is involved in formation of telomeric heterochromatin, resulted in spreading of regions in which lysine 79 was hypomethylated, whereas disruption of heterochromatin by Sir3 deletions resulted in increased lysine 79 methylation. High lysine 79 methylation levels were also found at non-heterochromatic sites that contained either actively transcribed or inactive genomic loci. These results support the idea that lysine 79 hypomethylation is closely associated with heterochromatin and that hypermethylated lysine 79 serves as a marker for euchromatin.

In order to extend this observation to mammalian species, the authors [6] examined immunoglobulin and TCR loci in murine progenitor $\mathrm{B}$ - and T-cell lines derived from mice lacking the RAG recombinase. Because the recombinase cannot be assembled in these cells, they are developmentally arrested before V(D)J rearrangement and serve as models for the regulation of chromatin accessibility. Lysine 79 methylation and lineage specificity was found to be correlated: B cells, which have the potential to rearrange immunoglobulin but not TCR genes, showed elevated lysine 79 methylation at an immunoglobulin but not a TCR locus, whereas a reciprocal pattern was detected in T cells. Furthermore, the lysine 79 methylation pattern reflected developmental stages: $\mathrm{B}$ and $\mathrm{T}$ cells that were arrested before rearrangement of $\mathrm{D}$ and $\mathrm{J}$ segments, which are combined first, had high lysine 79 methylation at $\mathrm{D}$ and $\mathrm{J}$ regions, but not at their $\mathrm{V}$ regions, which are recombined with the joint DJ segment later. Thus, methylation of lysine 79 precedes V(D)J rearrangement and may be important for promoting access to recombination signal sequences.

How is lysine 79 methylation regulated? Dot1 methylated lysine 79 in S. cerevisiae, and mammalian homologs of Dot1 have been identified $[1,16]$. In contrast to most of the other 
known histone methyltransferases, Dot1 does not methylate isolated histones but prefers nucleosomal substrates; thus, other histone modifications are expected to influence Dot1 activity. Indeed, ubiquitination of histone H2B at lysine 123 is required for methylation of histone $\mathrm{H}_{3}$ lysine 79 in vitro (reviewed in [1]). In addition, methylation of lysine 79 of histone $\mathrm{H}_{3}$ shows an inverse relationship with acetylation of lysine 16 of histone $\mathrm{H}_{4}$ [6], and hypomethylation of lysine 79 of histone $\mathrm{H}_{3}$ depends on the presence of Sir proteins, because Sir proteins preferentially interact with unmethylated lysine 79 and block further Dot1-mediated methylation of this residue [6]. According to the authors' model [6], similar silencing proteins may play a role in murine cells, where they could create a chromatin structure that inhibits methylation by Dot1 homologs and thus prevent access of the RAG recombinase. Alternatively, lysine 79 methylation might prevent the association of silencing proteins with chromatin and promote Dot1 recruitment, stimulating additional lysine 79 methylation and recombinase accessibility.

The accessibility hypothesis has been nourished over the last few years by studies that revealed correlations between a number of different histone modifications and the recombinationally active state of specific genes. The data support the idea that a histone code determines accessibility of chromatin to proteins that ultimately controls V(D)J recombination. What is the future outlook? We do not yet know how the various chromatin-modifying activities are recruited to specific cis-acting enhancer elements, but undoubtedly additional novel histone modifications will be identified as being closely associated with controlling access of recombination factors to DNA. Because many of the findings to date are correlative, we will have to discriminate in future which histone modifications are truly crucial for regulating access. It will be challenging to study the cascade of events that ultimately leads to the final chromatin structure that is required for recombinase access. If a histone code serves as a platform for the recruitment of other chromatin-associated complexes and proteins, such as SWI/SNF complexes, heterochromatin protein 1 (HP1) or silencing proteins, how will these factors fit into the cascade of events that regulates access for RAGmediated cleavage? Finally, will there be a universal histone code identified that defines all recombination-competent sites, and will this code be distinct from the code that defines transcriptional competence? The uniqueness of the V(D)J recombination process, which can cause serious disease when defective, might demand a unique control mechanism. To date, no histone modifications specific to V(D)J recombination sites have been identified, but the search is still on.

\section{Acknowledgements}

We thank Joost Oppenheim and Michael Krangel for critical comments on the manuscript. The content of this publication does not necessarily reflect the views or policies of the Department of Health and Human Services, nor does mention of trade names, commercial products, or organizations imply endorsement by the US Government. This project has been funded in whole or part with Federal funds from the National Cancer Institute, National Institutes of Health, under Contract No. NOI-C0-I2400.

\section{References}

I. Turner BM: Cellular memory and the histone code. Cell 2002, I I I:285-29I.

2. Grewal SI, Elgin SC: Heterochromatin: new possibilities for the inheritance of structure. Curr Opin Genet Dev 2002, I 2: I78187.

3. Yancopoulos GD, Alt FW: Developmentally controlled and tissue-specific expression of unrearranged $\mathrm{VH}$ gene segments. Cell 1985, 40:27I-28I.

4. Roth DB, Roth SY: Unequal access: regulating $V(D) J$ recombination through chromatin remodeling. Cell 2000, I 03:699-702.

5. Bassing $\mathrm{CH}$, Swat $\mathrm{W}$, Alt FW: The mechanism and regulation of chromosomal V(D)J recombination. Cell 2002, I 09 Suppl:S4555.

6. $\mathrm{Ng} \mathrm{HH}$, Ciccone DN, Morshead KB, Oettinger MA, Struhl K: Lysine-79 of histone $\mathrm{H} 3$ is hypomethylated at silenced loci in yeast and mammalian cells: $A$ potential mechanism for position-effect variegation. Proc Natl Acad Sci USA 2003, 100:1820-1825.

7. Krangel MS : V(D)J recombination becomes accessible. J Exp Med 200I, 1 93:F27-30.

8. Hsieh CL, Gauss G, Lieber MR: Replication, transcription, CpG methylation and DNA topology in V(D)J recombination. Curr Top Microbiol Immunol 1992, I 82: I 25- I 35.

9. Cherry SR, Beard C, Jaenisch R, Baltimore $D$ : V(D)J recombination is not activated by demethylation of the kappa locus. Proc Natl Acad Sci USA 2000, 97:8467-8472.

10. Senoo M, Mochida N, Wang L, Matsumura Y, Suzuki D, Takeda N, Shinkai $Y$, Habu S: Limited effect of chromatin remodeling on $\mathrm{D}$ (beta)-to-J(beta) recombination in CD4+CD8+ thymocyte: implications for a new aspect in the regulation of TCR beta gene recombination. Int Immunol 200I, I3:I 405-I4I4.

II. Sikes ML, Meade A, Tripathi R, Krangel MS, Oltz EM: Regulation of $V(D) J$ recombination: a dominant role for promoter positioning in gene segment accessibility. Proc Natl Acad Sci USA 2002, 99: |2309-12314.

12. Su IH, Basavaraj A, Krutchinsky AN, Hobert O, Ullrich A, Chait BT, Tarakhovsky A: Ezh2 controls B cell development through histone $\mathbf{H 3}$ methylation and Igh rearrangement. Nat Immunol 2003, 4:|24-|3|.

I3. Huang J, Muegge K: Control of chromatin accessibility for V(D)J recombination by interleukin-7. J Leukoc Biol 200I, 69:907-91।.

14. Strahl BD, Allis CD: The language of covalent histone modifications. Nature 2000, 403:4I-45.

15. Park JH, Cosgrove MS, Youngman E, Wolberger C, Boeke JD: A core nucleosome surface crucial for transcriptional silencing. Nat Genet 2002, 32:273-279.

16. Varga-Weisz PD, Dalgaard JZ: A mark in the core: silence no more! Mol Cell 2002, 9: I I54-I I56.

17. $\mathrm{Ng} \mathrm{HH}$, Feng Q, Wang H, Erdjument-Bromage H, Tempst P, Zhang $Y$, Struhl K: Lysine methylation within the globular domain of histone $\mathbf{H} 3$ by Dot I is important for telomeric silencing and Sir protein association. Genes Dev 2002, I6:1518-1527. 